IAU Colloquium 164: Radio Emission from Galactic and Extragalactic Compact Sources

ASP Conference Series, Vol. 144, 1998

J. A. Zensus, G. B. Taylor, \& J. M. Wrobel (eds.)

\title{
New Radio Observations of Circinus X-1: A Summary
}

\author{
R. P. Fender \\ Astronomy Centre, University of Sussex, Brighton BN1 9QH U.K.
}

\begin{abstract}
New radio observations from the Australia Telescope Compact Array of the radio jet X-ray binary Circinus X-1 are summarized. Radio flux density monitoring around nearly an entire orbit is presented, confirming continued radio flaring around phase 0.1 (near periastron passage) as well as a lesser enhancement half an orbit later.
\end{abstract}

\section{Introduction}

Circinus $\mathrm{X}-1$ is an unusual bright and variable $\mathrm{X}$-ray source. The system is also radio bright, though having faded since the 1970 s when it used to reach peak flux densities in excess of $1 \mathrm{Jy}$ (e.g., Haynes et al. 1978). Once every 16.6 days, the source undergoes radio, infrared and X-ray activity, indicative of a heightened accretion rate. This is generally interpreted as periastron passage of a neutron star in an elliptical 16.6 day orbit about its companion.

The source is embedded within a synchrotron nebula, from which there is evidence of emission trailing back towards the nearby supernova remnant G321.9-0.3. Furthermore, ATCA maps at $6.3 \mathrm{~cm}$ (Stewart et al. 1993) suggest the appearance of swept-back jet-like structures extending from the core of Cir X1 out into the nebula. They suggest that the source has radio jets c.f. SS 433 which are swept back due to a high velocity through the ISM away from its birthplace in G321.9-0.3.

\section{Observations and Results}

Cir X-1 is a very important system for the study of radio jets from X-ray binaries, being the only one of these objects for which there is both good evidence for the existence of a jet and direct evidence that the compact object is a neutron star (from observations of Type I X-ray bursts). However, the declining radio brightness since the 1970s makes Cir X-1 hard to observe at HartRAO (Nicolson, priv. comm.), and observations from MOST at $843 \mathrm{MHz}$ are swamped by emission from the surrounding synchrotron nebula. As a result, the observations reported here are the first of this source for several years.

In 1996 July we observed Cir X-1 with the ATCA at $6.3 \& 3.5 \mathrm{~cm}$ on 10 days over $\sim 14$ days of its 16.6 -day orbit; the $6.3 \mathrm{~cm}$ flux density, as a function of phase, is plotted in Fig 1. The radio variability over the orbit is well correlated with soft X-ray monitoring from the RXTE ASM and this is discussed, along with a comparison of the new $6.3 \& 3.5 \mathrm{~cm}$ radio data, in Fender (1997).

Mapping of the new data, in particular from the first two runs, which were over 12 and $10 \mathrm{hr}$ respectively, reveals some hints of extended structure at a similar position angle to that of the jet presented in Stewart et al. (1993). However, uncertainties in the contribution to the synthesised maps both from intrinsic source variability and the synchrotron nebula in which the source is embedded make confident interpretation difficult. 


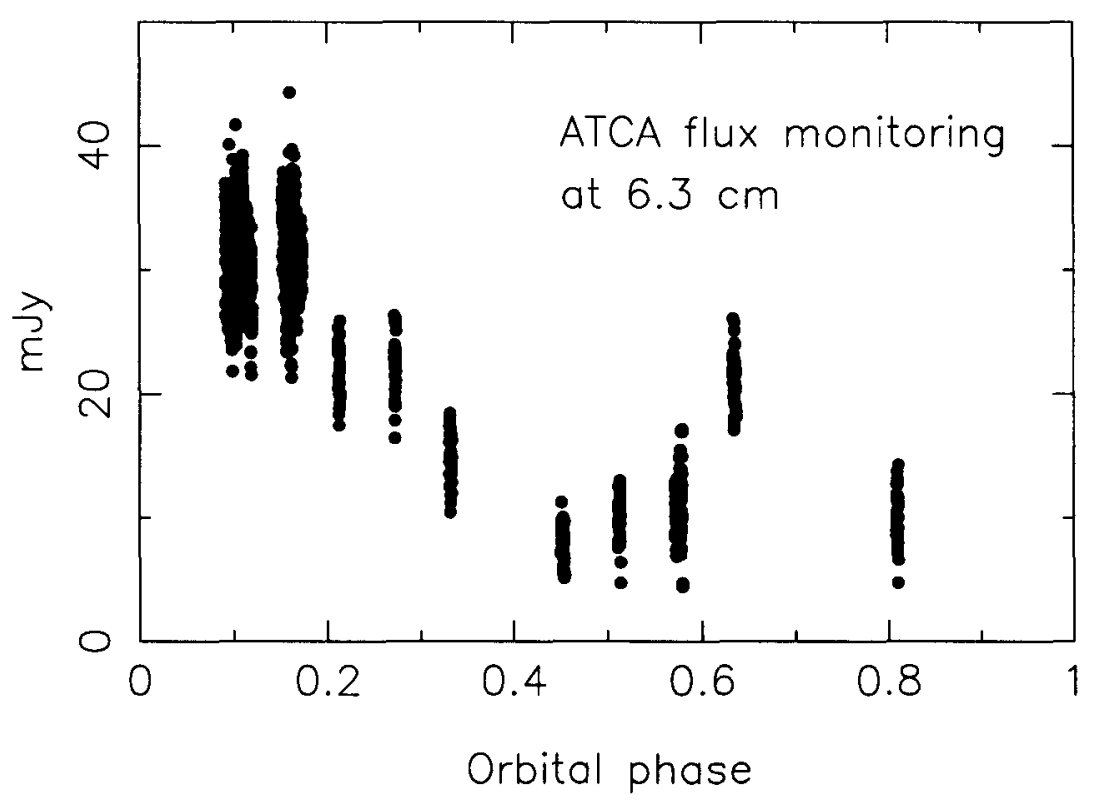

Figure 1. Flux monitoring of Cir X-1 at $6.3 \mathrm{~cm}$ with ATCA in $1996 \mathrm{July}$ over most of its 16.6-day orbit. The $3.5 \mathrm{~cm}$ flux monitoring, not plotted here, tracks the $6.3 \mathrm{~cm}$ fluxes closely. Note, as well as the enhanced fluxes around phase 0.1 (near periastron passage), significant brightening half an orbit later.

While lack of space precludes an in-depth discussion of the new data, some important points should be pointed out;

- The source continues to undergo radio flaring between phases 0 and 0.2 .

- The secondary flaring observed around phase 0.6 has not been previously reported. However, this is correlated with an increase in X-ray activity at this phase (see Fender [1997] for a fuller discussion of X-ray : radio behavior).

- The source is significantly stronger in these observations than during the observations of Stewart et al. (1993). While still far below the level observed in the 1970s (e.g., Haynes et al. 1978), this may indicate a gradual brightening over the past five years or so.

Acknowledgments. I thank Karen Southwell and Vince Mclntyre for assistance with the observations, and thank Jim Caswell, Bob Sault, Tasso Tzioumis and Kinwah Wu for useful discussions. The ATCA is funded by the Commonwealth of Australia for operation as a National Facility managed by CSIRO.

\section{References}

Fender, R. P, 1997, in Proc. 4th Compton Symposium, AIP, in press.

Haynes, R. F., et al., 1978. MNRAS, 185, 661-671.

Stewart, R. T., et al., 1993. MNRAS, 261, 593-598. 